\title{
Systèmes à tension de vapeur non négligeable : diagrammes T-v et $\mathrm{p}-\mathrm{T}$ de l'adamantane
}

\author{
P. Espeau et R. Céolin
}

Laboratoire de Chimie Physique, Université Paris V, Faculté des Sciences Pharmaceutiques

et Biologiques, 4 avenue de l'Observatoire, 75006 Paris, France

\begin{abstract}
A DSC method, designed to determine the melting enthalpy of compounds with non-negligible vapour pressure, is applied to adamantane. This method, based upon the linear dependence of the melting-related thermal effect with the specific volume $v$, is used to construct the T-v diagram of adamantane through which a melting

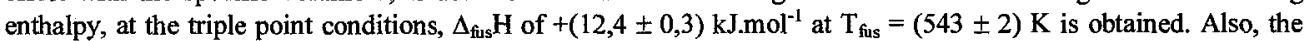
specific volume of the vapour phase is extrapolated to $(52,7 \pm 1,5) \mathrm{cm}^{3} \cdot \mathrm{g}^{-1}$. With this information, a more general description of the $\mathrm{p}-\mathrm{T}$ diagram for adamantane is put forward.
\end{abstract}

\section{INTRODUCTION}

Une méthode précédemment utilisée pour déterminer l'enthalpie de fusion de l'arsenic [1] est ici appliquée au cas de l'adamantane.

L'adamantane (tricyclo[3.3.1.1 $1^{3,7}$ ]décane), $\mathrm{C}_{10} \mathrm{H}_{16}$, subit, à $\mathrm{T}=208,6 \mathrm{~K}$ [2], une transformation d'une phase quadratique $S_{\text {II }}\left(P \overline{4} 2_{1} \mathrm{c}, \mathrm{a}=6,641 \AA\right.$ et $\mathrm{c}=8,875 \AA$ à $\mathrm{T}=208,6 \mathrm{~K}$ [3]) vers une phase plastique cubique $\mathrm{S}_{\mathrm{I}}(\mathrm{Fm} 3 \mathrm{~m}, \mathrm{a}=9,42 \AA \AA$ à $293 \mathrm{~K}$ [4]) avant de fondre à $543 \mathrm{~K}$.

Les analyses DSC ont été effectuées à l'aide d'un analyseur thermique TA 2000 (TA Instruments) et d'un DSC7 (Perkin-Elmer), à une vitesse de $5 \mathrm{~K} / \mathrm{min}$. Les creusets en acier haute pression Setaram et Perkin-Elmer (de volume interne de $30 \mu \mathrm{L}$ ) ont été remplis avec des masses d'adamantane allant de 0,67 et $26,97 \mathrm{mg}$.

\section{METHODE}

Le diagramme $\mathrm{T}-v$ présenté à la Figure 1 montre comment le point triple dans une représentation $\mathrm{p}-\mathrm{T}$ devient un palier horizontal dans le plan $\mathrm{T}-\boldsymbol{v}$. Un diagramme de Tammann peut alors être associé au "segment triple" A-E-B.

En considérant le rapport $\mathrm{V} / \mathrm{m}=v$ entre le volume interne total de la capsule DSC et la masse d'échantillon, i.e. le volume spécifique de la matière hétérogène, l'enthalpie de fusion peut alors être extrapolée à $v_{\mathrm{L}}$ (i.e. la valeur de $v$ au point $\mathrm{E}$ ). Les points $\mathrm{A}, \mathrm{E}$ et $\mathrm{B}$ correspondent respectivement aux volumes spécifiques des phases solide, liquide et vapeur aux conditions du point triple. 

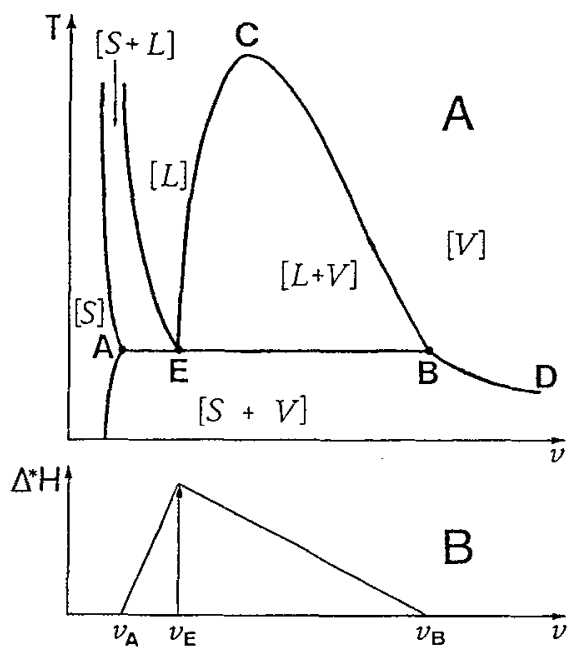

Figure 1 : A : représentation schématique dans le plan $T-v$ d'un point triple,

$\mathrm{B}$ : diagramme de Tammann associé au segment AEB

\section{RESULTATS ET DISCUSSION}

\subsection{Diagramme T-v}

Trois types de courbes DSC sont obtenus en fonction du rapport V/m (Figure 2) au voisinage du point triple :

A- A faible $\mathrm{V} / \mathrm{m}$ : le segment EB est traversé.

B- A V/m suffisamment grand : la courbe $B C$ de la Figure 1 est traversée après le palier EB.

C- A V/m très grand $\left(>\right.$ à $\left.52,7 \mathrm{~cm}^{3} \cdot \mathrm{g}^{-1}\right)$ : il n'y a plus de traversée du palier AEB, c'est la partie BD de la Figure 1 qui est franchie.
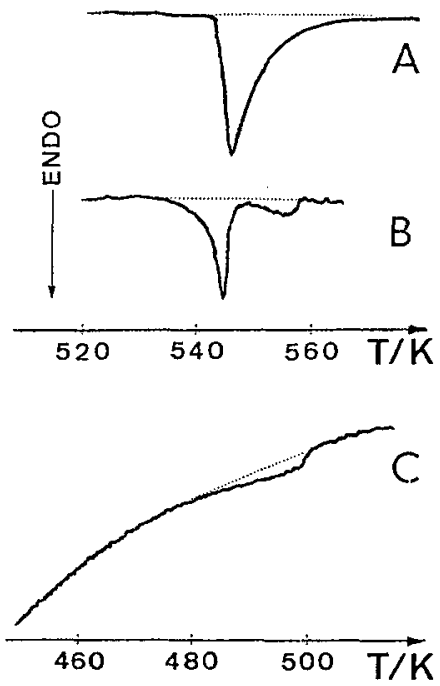

Figure 2 : Types de courbes DSC obtenues pour 3 valeurs différentes de V/m. 
Le résultat des 27 expérimentations DSC est représenté sur le diagramme T-v de la Figure 3. La température de fusion au point triple $\mathrm{S}_{\mathrm{I}}-L-V$ est de $(543 \pm 2) \mathrm{K}$. La courbe de Tammann de la Figure 4 correspond à l'effet thermique $\Delta^{*} H$ relatif à la traversée du segment E-B de la Figure 3. L'évolution linéaire de $\Delta^{*} \mathrm{H}$ en fonction de $\mathrm{V} / \mathrm{m}$ est représentée par :

$$
\Delta^{*} \mathrm{H}=-1,765 \times(\mathrm{V} / \mathrm{m})+93,080 \quad\left(\mathrm{r}^{2}=0,994\right)
$$

\section{T/K}

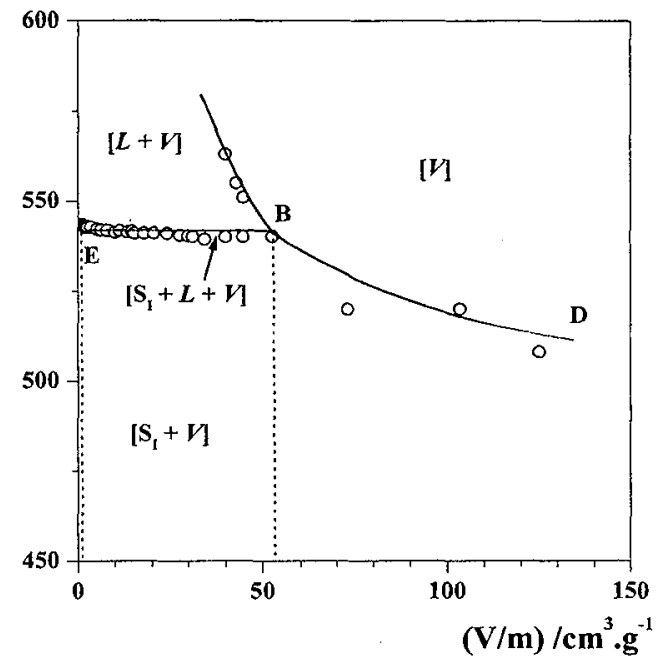

Figure 3 : Diagramme T-v de l'adamantane tracé à partir des points expérimentaux

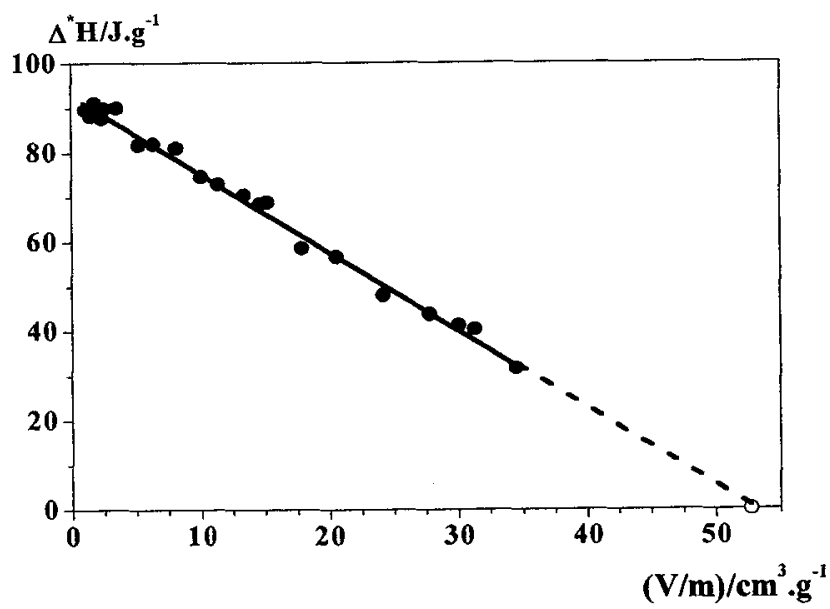

Figure 4 : Courbe de Tammann correspondant à la traversée du segment EB de la Figure 1 
Le volume spécifique $\left(v_{\mathrm{v}}\right)$ de la vapeur saturante à la température de fusion est obtenu pour $\Delta^{*} \mathrm{H}=0$ (point $\mathrm{B}$ ) et a été trouvé, d'après l'équation (1), égal à $52,7 \mathrm{~cm}^{3} \cdot \mathrm{g}^{-1}$. Ceci conduit à une masse molaire de $150,54 \mathrm{~g} \cdot \mathrm{mol}^{-1}$ (au lieu de 136,24 g. $\mathrm{mol}^{-1}$ ), assumant que l'adamantane se comporte comme un gaz parfait et que la pression au point triple est $569048 \mathrm{~Pa}$ (d'après l'équation 5 du tableau 1). L'enthalpie de fusion, $\Delta_{\text {fus }} \mathrm{H}$, peut être calculée au point $\mathrm{E}$ à partir de l'équation (1) en déterminant, au préalable, le volume spécifique du liquide au point $\mathrm{E}\left(v_{L}\right)$ d'après $[5,6], v_{\mathrm{L}}=1,056 \mathrm{~cm}^{3} \cdot \mathrm{g}^{-1}\left(143,9 \mathrm{~cm}^{3} \cdot \mathrm{mol}^{-1}\right)$. Le calcul donne $\Delta_{\text {fus }} \mathrm{H}=+91,20 \mathrm{~J} \cdot \mathrm{g}^{-1}\left(+12,43 \mathrm{~kJ} \cdot \mathrm{mol}^{-1}\right)$.

\subsection{Diagramme p-T}

L'existence de deux polymorphes $\left(\mathrm{S}_{\mathrm{I}}\right.$ and $\left.\mathrm{S}_{\mathrm{II}}\right)$ de l'adamantane entraîne celle de quatre points triples : $\mathrm{S}_{\mathrm{I}}-L-V$ (point 1 ), $\mathrm{S}_{\mathrm{I}}-\mathrm{S}_{\mathrm{I}^{-}}-V$ (point 2), $\mathrm{S}_{\mathrm{I}^{-}} \mathrm{S}_{\mathrm{II}}-L$ (point 3 ) et $\mathrm{S}_{\mathrm{II}}-L-V$ (point 4 ). Le tableau 1 résume l'ensemble des équations ayant servi à la construction du diagramme $\mathrm{p}-\mathrm{T}$, certaines de ces équations ayant été réajustées en tenant compte de la valeur $\Delta_{\text {fus }} \mathrm{H}=+12,43 \mathrm{~kJ} \mathrm{~mol}^{-1}$.

Tableau 1 : Equations des différents équilibres obtenus pour la construction du diagramme $\mathrm{p}-\mathrm{T}$.

\begin{tabular}{|c|c|c|c|}
\hline $\mathrm{N}^{\circ}$ eq. & Equilibres & Equations obtenues & Ref. \\
\hline $2^{*}$ & $\mathrm{~S}_{\mathrm{I}}-L$ & $\left.\mathrm{p} / \mathrm{kbar}=2,392 \times[(\mathrm{T} / \mathrm{K}) / 541)^{8,493}-1\right]$ & {$[5]$} \\
\hline $3^{* *}$ & $\mathrm{~S}_{\mathrm{I}}-L$ & $\mathrm{p} / \mathrm{Pa}=194,64 \times 10^{5} \times(\mathrm{T} / \mathrm{K})-11,752 \times 10^{9}\left(\mathrm{r}^{2}=0,989\right)$ & {$[5]$} \\
\hline $4^{* * *}$ & $\mathrm{~S}_{\mathrm{I}}-V$ & $\mathrm{p} / \mathrm{Pa}=20,054 \times 10^{10} \times \mathrm{e}^{-6935,5 /(\mathrm{T} / \mathrm{K})}\left(\mathrm{r}^{2}=0,997\right)$ & {$[7]$} \\
\hline 5 & $L-V$ & $\mathrm{p} / \mathrm{Pa}=1,277 \times 10^{10} \times \mathrm{e}^{-5440,2 /(\mathrm{T} / \mathrm{K})}$ & \\
\hline 6 & $\mathrm{~S}_{\mathrm{I}}-\mathrm{S}_{\mathrm{II}}$ & $\mathrm{p} / \mathrm{Pa}=\left(51 \times 10^{5} \times(\mathrm{T} / \mathrm{K})\right)-1,064 \times 10^{9}$ & {$[5,8,9]$} \\
\hline 7 & $\mathrm{~S}_{\mathrm{II}}-V$ & $\mathrm{p} / \mathrm{Pa}=14,033 \times 10^{11} \times \mathrm{e}^{-7341,4 /(\mathrm{T} / \mathrm{K})}$ & \\
\hline 8 & $\mathrm{~S}_{\mathrm{II}}-L$ & $\mathrm{p} / \mathrm{Pa}=8,04 \times 10^{6} \times(\mathrm{T} / \mathrm{K})-3,253 \times 10^{9}$ & \\
\hline
\end{tabular}

${ }^{*}:$ courbe obtenue pour des pressions allant jusqu'à $25 \mathrm{kbars}$.

**: Ajustement à partir des valeurs expérimentales données dans [5].

*** : Paramètrage obtenu à partir des valeurs recommandées par [7], 6935,5 est le rapport entre l'enthalpie de sublimation et la constante $R$ du gaz parfait.

Ceci conduit à $\Delta_{\text {sub }} \mathrm{H}=+57,66 \mathrm{~kJ} \cdot \mathrm{mol}^{-1}$ et à $\Delta_{\text {vap }} \mathrm{H}=+45,23 \mathrm{~kJ} \cdot \mathrm{mol}^{-1}$, à $543 \mathrm{~K}$, d'après : $\Delta_{\text {sub }} \mathrm{H}\left(\mathrm{S}_{\mathrm{I}}\right)=\Delta_{\text {fus }} \mathrm{H}+\Delta_{\text {vap }} \mathrm{H}$.

On peut alors calculer l'enthalpie de sublimation de la phase $\mathrm{S}_{\mathrm{II}}$ :

$\Delta_{\text {sub }} \mathrm{H}\left(\mathrm{S}_{\mathrm{II}}\right)=\Delta_{\text {sub }} \mathrm{H}\left(\mathrm{S}_{\mathrm{I}}\right)+\Delta_{\text {trans }} \mathrm{H}\left(\mathrm{S}_{\mathrm{II}} \rightarrow \mathrm{S}_{\mathrm{I}}\right)$ avec $\Delta_{\text {sub }} \mathrm{H}\left(\mathrm{S}_{\mathrm{I}}\right)=+2,75 \mathrm{~kJ} \mathrm{~mol}^{-1}$ d'après [8].

Ainsi : $\Delta_{\text {sub }} H\left(\mathrm{~S}_{\mathrm{II}}\right)=+61,04 \mathrm{~kJ} \cdot \mathrm{mol}^{-1}$.

L'ensemble de ces résultats permet de tracer le diagramme $\mathrm{p}-\mathrm{T}$ représenté sur la Figure 5. L'intersection de ces courbes prises trois à trois donne la position des quatre points triples ( 3 stables et un métastable) répertoriés dans le tableau 2. 
Ce diagramme est topologiquement identique à celui du dimorphisme du soufre comportant trois points triples stables et un point triple métastable.
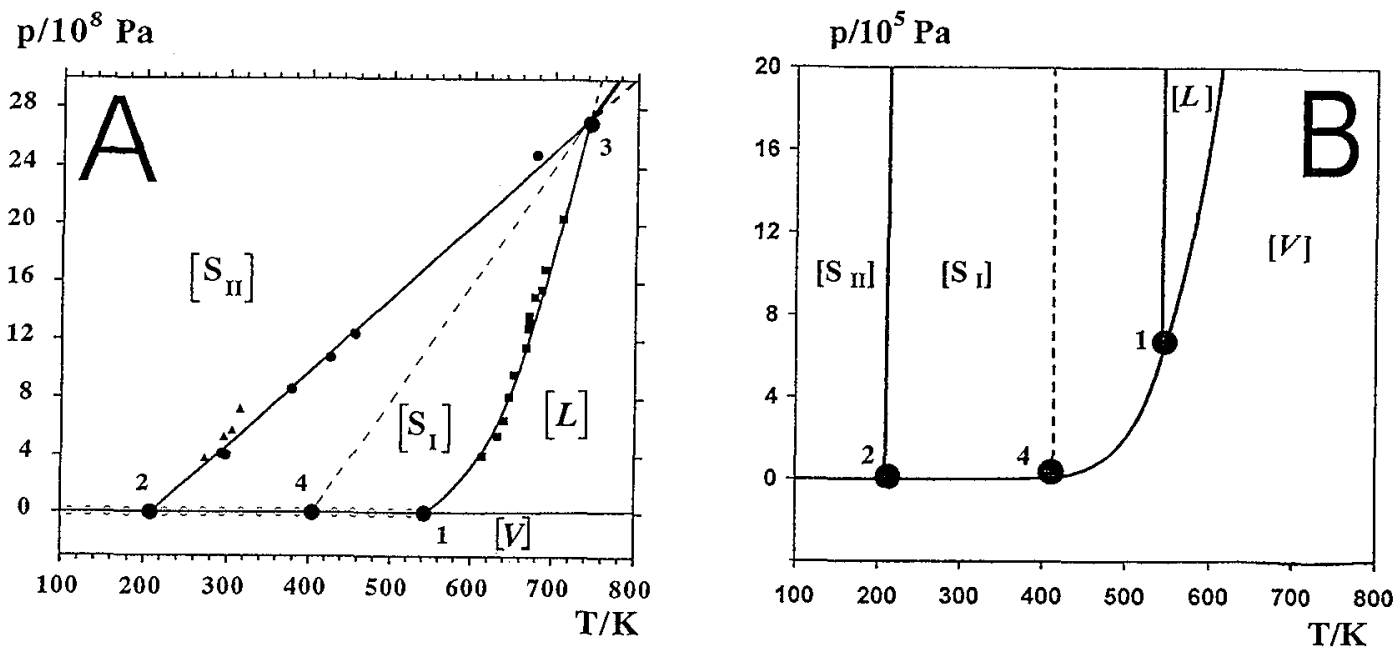

Figure 5 : A : Représentation du diagramme expérimental p- $T$ de l'adamantane avec localisation des 4 points triples.

Points expérimentaux : $\mathbf{\square}$ d'après [5], d'après [8], $\Delta$ d'après [9], $O$ d'après [7]

B : Agrandissement de la partie basse pression.

(les équilibres métastables sont représentés en pointillés).

Tableau 2 : Coordonnées approchées des quatre points triples dans le diagramme p-T

\begin{tabular}{|l|c|c|}
\hline & $\mathrm{T} / \mathrm{K}$ & $\mathrm{P} / \mathrm{Pa}$ \\
\hline $\mathrm{S}_{\mathrm{I}}-L-V \quad(1)$ & 543 & $5,7 \times 10^{5}$ \\
\hline $\mathrm{S}_{\mathrm{I}}-\mathrm{S}_{\mathrm{II}}-V(\mathbf{2})$ & 209 & $73 \times 10^{-5}$ \\
\hline $\mathrm{S}_{\mathrm{I}}-\mathrm{S}_{\mathrm{II}}-L$ (3) & 744 & $27,3 \times 10^{8}$ \\
\hline $\mathrm{S}_{\mathrm{II}}-L-V$ (4) & 405 & $19 \times 10^{3}$ \\
\hline
\end{tabular}

$\mathrm{S}_{\mathrm{II}}-L-V$ est le point triple métastable.

\section{Références}

[1] Rouland J. C., Céolin R., Souleau C. and Khodadad P., J. Thermal Anal. 23 (1982) 143-151.

[2] Chang S.S. and Westrum Jr. E.F., J. Phys. Chem. 64 (1960) 1547-1551.

[3] Mirskaya K.V., Kristallografiya, 8 (1963) 225.

[4] Tonkov E. Yu., High Pressure Phase Transformations Handbook, vol. 1 (Gordon and Breach

Science Publishers, Philadelphia, 1992) pp. 156-157.

[5] Pistorius C.W.F.T. and Resing G.C., Mol. Cryst. Liq. Cryst. 5 (1969) 353-361.

[6] Amoureux J.P., Bee M. and Damien J.C., Acta Cryst. B36 (1980) 2633-2636.

[7] Mokbel I., Rúzicka K., Majer V., Rúzicka V., Ribeiro M., Jose J. and Zábransky M., Fluid Phase Equilibria 169 (2000) 191-207.

[8] Pistorius C.W.F.T. and Snyman H.C., Z. Phys. Chem. 43 (1964) 278-281.

[9] Hara K., Katou Y. and Osugi J., Bull. Chem. Soc. Jpn. 54 (1981) 687-691. 